Plummer's figures it appears that in longitude $223^{\circ} \cdot 4$ the comet is distant from the orbit of Jupiter only $0^{\prime} 145$, a sufficient explanation of a probable cause of the short period of revolution.

THE Great COMET of 1882. - The positions subjoined are extracted from an ephemeris published by Herr Stechert, of Berlin (Astron. Nach. No. 2486), and founded upon the elliptical elements of Dr. Kreutz:-

\section{At Berlin Midnight}

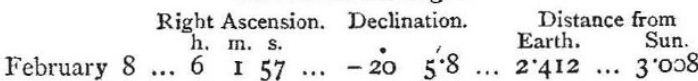

$$
\begin{aligned}
& \begin{array}{lllllllll}
10 & \ldots & 6 & 0 & 17 & \ldots & 19 & 34^{\circ} 5
\end{array} \\
& \begin{array}{llllllllllllll}
12 & \ldots & 5 & 5^{8} & 46 & \ldots & 19 & 3.7 & \ldots & 2.506 & \ldots & 3.053
\end{array} \\
& \begin{array}{lllllllllll}
14 & \ldots & 5 & 57 & 25 & \ldots & 18 & 33.4
\end{array} \\
& \begin{array}{llllllllllll}
16 & \ldots & 5 & 57 & 25 & \ldots & 18 & 33 & 4 & & & \\
16 & \ldots & 5 & 5 & 12 & \ldots & 18 & 3.6 & \ldots & 2 \cdot 602 & \ldots & 3.118
\end{array} \\
& \begin{array}{llllllllll}
18 & \ldots & 5 & 55 & 8 & \ldots & 17 & 34^{\circ} 4
\end{array} \\
& \begin{array}{lllllllllllll}
20 & \ldots & 5 & 54 & 1 & \ldots & -17 & 57 & \ldots & 27 & 700 & \ldots & 31172
\end{array}
\end{aligned}
$$

Astronomical Telegrams.-Mr. Spencer F. Baird, Secretary of the Smithsonian Institution, notifies that arrangements have been completed with the Director of the Harvard College Observatory for conducting the system of telegraphic announcements of astronomical discoveries, which was established by the Institution in 1873 , and that henceforward the American centre of reception and distribution of telegrams will be "The Harvard College Observatory, Cambridge, Massachusetts," to which all astronomical telegrams should in future be sent.

\section{THE MATTER OF SPACE}

$\mathrm{F}$ late years there has been a growing tendency towards the belief that matter is present everywhere throughout the universe, as well in interstitial space as in the bodies of the spheres. Yet an older hypothesis is still widely held. The phenomena of light seem to require some substantial medium in space, but this substance has been viewed as specifically distinct fron matter, and named ether. Another class of thinkers has devised stil another species of substance. This is required to meet the demands of the new gravitation hypothe.is; and consists of excessively minute particles, moving with intense speed, and pressing vigo:ously on the larger and slower particles of matter. In the past still other species of substance were imagined; heat, electricity, etc., were each ascribed to a specifically distinct substance.

Now, however, the tide has turned, and the inclination is to believe in only a single form of substance. There are, of course, countless distinct conditions produced by the aggregations of substance, and variations from simplicity to co nplexity, but thi; may not necessarily require more than a single kind of basic particle, or whatever we may call it. If the substantial contents of space are similar in constitution to the matter of the spheres, their state of existence must be much more simplified. In the spheres we have matter ranging from the simple elementary gases of the atmosphere, through the complex mineral compounds of the solid surface, to the highly compounded organic molecules. In outer space the variation is probably in the opposite direction, and substance may exist there in a condition much more highly disintegrated thin the atmospheric gases. This view is not held by all theorists. Dr. Siemens argues that space holds molecules of considerable intricacy, comprising certain terrestrial elements, and their simpler compounds; as to the contents of space we know that there are very numerous solid masses, some of considerable size, others minute, and pussibly ranging throush many degrees from the largest $t s$ the minutest. Yet these really occupy but an inconsiderahle portion of space, and apparently originated in s slar or planetary orbs.

Such is, briefly stated, the state of knowledge and of hypothesis concerning the substantial contents of space. We need but add the uncertain reasons for arguing the presence of a resisting medium in space, and the necessity of a highly elastic condition of the light-conducting substance, to exhaust the subject so far as yet pursued.

It is held by some that the gravitation energy of the suns and planets is sufficiently great to sweep space of all contiguous material particles, except those solid masses which are saved from this fate by the vigour of their orbital motisns. The atmospheres of suns and planets are retained with an energy very greatly in exces; of their reverse energy of molecular motim, and therefore it is quite impossible that any of this material should escape into space, or that any similarly-conditioned material should exist contiguous to the spheres without being forced to become atmospheric matter. The centrifugal energy of the earth's atmosphere at the equator is only ${ }_{17}^{1}$ of that necessary to overcome gravity. The molecules of the atmosphere have also a vigour of heat vibration absut equal to their centrifugal energy. Hence the resisting energy of these molecules is far below the gravitative energy, and they are vigorously held.

The question of the possible existence of gravitating matter in interspheral space depends strictly upon that of its motor energy. If the momentum of any particle, or of the whole sum of particles, be insufficient to constituie a centrifugal energy equal or superior to the centripetal energy of gravitation, then the material contents of space must inevitably be drawn into the attracting spheres, as atmospheric substance, and space be denuded of matter. If, on the contrary, the centrifugal energy of these particles be sufficient to resist gravitation, they will remain free, and space continue peopled by matter.

Such gravitative particles, wherever existing in space, could not be for an instant free from the influence of spheral attraction whatever their energy of motion. If this energy be too small, they must be related to the spheres as falling bodies, and must become atmospheric matter. If the two opposite energies be equal, they must be related to the spheres as planetary bodies, and circle in fixed orbits around the centre of attraction. If the centrifugal energy be in excess they must assume the condition of independent cometary bodies, temporarily influenced but not permanently contrulled by any sun, and wandering eternally through space.

Such are the three possible conditions of the material contents of space. If the first obtain, space must be denuded of matter; if the second obtain, it will permanently contain matter in a partially elastic state; if the third obtain, it will permanently contain matter in a highly elastic state, since the pressure upon each other of the vigorously centrifugal particles must be great, and may be extreme. Of course no single particle could long retain its direction of motion, as related to any sphere. Constant impacts must constintly vary the directions of molecular motion. But the motion of each particle is successively transferred to a long series of particles, and thus is virtually continued in force and direction. Each motion pursues its course independently, though not as affecting any fixed particle of matter, and each particle aids in the progression of a vast network of motions, proceeding in every directi on throughout the universe. Thus each particle, though not actually changing its place, may have motor relations which extend in every direction to the utmost extremes of space. It is a node in ant interminable network of motions, and its incessant leaps throughout the limits of its narrow space are each part of a long motor line, which affects successively myriads of parti.iles. So far as the energy of gravitation is concerned the effect upon this incessantly transferred motion is precisely the same as if the motion was confined to a single particle. If it lack energy the motion will be a falling one; if it equal the gravitative energy it will form a closed orbit. If it exceed the gravitative energy it will form an open curve, and be only temporarily controlled by any sphere.

In this interchange of motor energy certain particles may continually decrease in vigour of motion, and if near solar orb may be drawn in as atmospheric matter. But they can only lose motion by transferring it to others, which would in consequence become more independent of gravity. The sum of motor energies in the universe must persist unchanged, and the aggregation of atmospheric substance around any planet must cause an outflow of motor energy which will increase the motor vigour of exterior particles. In such a case the height of atmosphere in any sphere will depend, partly on the attractive vigour of the sphere, and partly on the average motor vigour of the whole sum of matter. Every contraction and loss of motor energy by any portion of matter will increase the motor energy of remaining matter, and a fixed limit to the atmospheric control of every sphere must result, since in the outer layers of its atmosphere the centrifugal energy of molecular motion must increase until it equals the energy of gravitation.

Can we arrive at any conclusion as to which of the three possible conditions above considered really exists? If so we can answer the question as to the existence of matter as a constant tenant of space, and also reach some conclusions as to the character of its motor conditions. 
There is one line of thought which seems to lead to a settlement of this question. If the nebular hypothesis of the formation of solar systems be accepted as true, either wholly or partly, there can be no doubt as to the interspheral status of matter. The conditions of nebular aggregation indisputably settle it.

This hypothesis holds that the matter now concentrated into suns and planets was once more widely disseminated, so that the substance of each sphere occupied a very considerable extent of space. It even declares that the matter of the solar system was a nebulous cloud, extending far beyond the present limits of that system. From this original condition the existing condition of the spheres has arisen, through a continued concentration of matter. But this concentration was constantly opposed by the heat energy of the particles, or, in other words, by their centrifugal momentum. This momentum could be only got rid of by a redistribution of motor energy. If, for illustration, the average momentum of the particles of the nebula was just equivalent to their gravitative energy, then a portion of this energy must radiate or be conducted outwards ere the internal particles could be held prisoners by gravitation. The loss of momentum inwardly must be correlated with an increase of momentum outwardly.

This is a necessary consequence of the heat relations of matter. As substance condenses its capacity for heat decreases, and its temperature rises, hence a difference of temperature must constantly have arisen between the denser and the rarer portions of the nebulous mass, and equality of temperature could be restored only by heat radiation. This radiation still continues, and must continue until condensation ceases, and the temperatures of the spheres and space become equalised, but this is equivalent to declaring that as the particles of the spheres decrease in heat momentum those of interspheral space increase, and if originally the centrifugal and centripetal energies of matter approached equality, they must become unequal, centripetal energy becoming in excess in spheral matter, centrifuyal energy in the matter of space. Thus, as a portion of the originally widely distributed nebulous matter lost its heat, and became permanenily fixed in place by gravitative attraction, another portion gained heat, became still more independent of gravity, and assumed a state of greater nebulous diffusicn than originally. The condensing spheres only denuded space of a portion of the matter which it formerly held, and left the remainder more thinly distributed than before. The spheres, in their concentration, have emitted, and are emitting, a vast energy of motion. This motor energy yet exists in space as a motion of the particles of matter, which therefore press upon each other, or seek to extend their limits, with increasing vigour, so that the elasticity of jinterspheral matter is constantly increasing.

It might be hastily imagined that such an excess of heat vigour in the matter of space over that of the spheres should declare itself in temperature. But it must be remembered that temperature is no measure of the absolute heat contents of matter.

Condensation increases, rarefaction decreases, temperature with no necessary change in absolute heat contents. The expression "fire mist," so of ten applied to the matter of uncondensed nebula, gives a very erroneous impression. The matter of the solar system nebula, though containing a high degree of absolute heat, was probably of low temperature. Its great rarity must certainly have greatly decreased its temperature. As a differentiation in this matter took place, one portion becoming condensed, another portion more rarefied, the former must have increased, the latter decreased, in temperature. Eventually the extreme condensation of one portion of this matter, and rarefaction of another, caused an extreme difference in temperature. An excessive radiation from the spheres to space has taken place in consequence, the absolute heat of the former constantly decreasing and that of the latter increasing. But the difference in temperature still continues great, the influence producing it acting much more rapidly than the influence tending to obliterate it. Eventually an equality of temperatures may be produced, but only by the production of a very considerable inequality of absolute heat. This must be the final result of spheral condensation and nebulous rarefaction of exterior matter; namely, equalization of temperature, with a change from the original homogeniety to a great heterngeniety of heat contents.

But we are again brought back to the question of the motor energies of matter. Are they sufficiently great to enable a portion of this matter, when reinforced in motor energy by radiations from the spheres, to defy gravitative attraction and, remain free in space? Undoubtedly so, and much greater than would be simply requisite for the purpose, since we find the matter of the planets, after their immense losses by radiation, still possessed of a considerable excess of motor energy. The earth, for instance, has an orbital motion sufficient to maintain it at a con:iderable distance from the sun. But the motion of the earth is but the combined motion of its molecules. This motion once existed as independent molecular motion, which in time, under the influence of gravity, became dependent molecular motion. We have already spoken of the fact that the particles of space, in consequence of their heat motions, tend to dart off in straight lines of motion, except in so far as the gravitative attraction of spheres causes these lines to become curved. These lines of motion, so far as individual particles are concerned, are checked by the particles coming into contact with others. The motion, however, proceeds onwards, though it is carried by successive, instead of by single particles. If, however, a number of particles move in company in the same direction, they may move much further as individuals, before transferring their energies. And if an immense mass of particles come to thus move in company their individual excursions may be indefinitely extended. The lines of motion, instead of being continued by successive particles, are continued by the same particles, and molecular motion becomes mass motion. The motion of terrestrial molecules, in their revolution around the sun, resemble those of the molecules in Prof. Brooks vacuum tubes, constituting his "fourth state of matter."

Now the degree of resistance of such a mass to centripetal energy will indicate the degree of resistance of the original uncombined molecules. In the earth the motion of the molecules, thus combined, yields a centrifugal energy sufficient to maintain the earth at its present distance from the sun. But this is only a portion of its molecular energies. Its molecules possess considerable independent motion, and form nodes in lines of radiation that extend in every direction. They have also lost a great vigour of motion by radiation to space. It follows that the original momentum of these molecules must have constituted a centrifugal vigour greatly in excess of their centripetal vigour. It secondarily follows that the momentum of those molecules of the nebula which still exist in space, augmented as it has been by radiations from the spheres, yields a very energetic excess of centrifugal vigour. Many of the comets have a centrifugal energy in excess of the centripetal energy of the sun, yet this represents only a fraction of the energy of their moleculec, and a much smaller fraction of the energy of the material particles of space.

The combination of the centrifugal energies of terrestrial particles is due to the fact of a secondary centre of gravity having been formed. The heat velocity of its particles, in excess of that displayed in their revolution around the sun, has become partly a revolution around the earth's axis, and is partly retained as heat vibration. But the heat velocity of the material particles of space is not thus secondarily employed. It is affected by the attraction of the sun, or of the nearest sphere; but evidently, from the considerations above taken, this attraction cannot be sufficient to over-balance the centrifugal energy and cause atmospheric aggregation or even to cause orbital revolution. The particles must have energy suff. cient to make them independent of spheral gravity. Their straight lines of motion must become to some degree curved in response to gravity, but cannot become closed curves. Instead of becoming planetary, they remain cometary lines, of very open orbit. For if we imagine the earth to be suddenly restored to its nebulous condition, or its particles to be set free in space, they would possess a ve'ocity of motion much in excess of the earth's orbital velocity. Hence they could not be controlled by the sun. The exi-ting particles of space possess a still much greater velocity, and are therefore much more free from gravitative control.

Certain necessary results of this condition have been considered. The lines of centrifugal motion in space are not con. fined to single particles as in the earth, but are transferred from particle to particle. The effect, however, is precisely the same; this motion of successive particles is in no respect different in effect from what we would have if a single particle were free to move in the same direction. Each particle moves a certain distance, and then transfers its motion in that direction to another. But it immediately pursues some other direction of motion in response to impact, and this aids in the progressive movement of innumerable lines of motor energy. The great centrifugal vigour of these motions must cause an energetic com. 
pressing infuence upon interspheral matter, and thus produce an elasticity, sufficient perhaps for the requirements of light radiation.

The lines of motion thus transferred through space cannot be unvarying in their orbital directions. Nature knows no great or small in her processes, and each movirg particle of the free matter of space is controlled by the same principles which control the motions of a planet. It is subject to perturbations from lateral attractions, similar to those which draw planets and comets out of their orbits, and completely cbange the orbit of the latter. And its impacts with other particles yield effects such as would arise in impacts between planets of oppositely moving systems. Action and reaction are equal, in this as in every case. The orbit and the speed of a line of motion may be changed through impact or attractive resistance, but only by its causing an opposite change in some other line. Taus the lines of motor energy referred to are not unvarying in speed and direction, but are unvarying in their sum of correlated speeds and directions. The variations which take place in the orbits of spheres and comets through attractive perturbation, and the greater variations which would take place did spheres come frequently into contact, are precisely similar to those which must occur in the case of interspheral particles, and any change in the direction of one orb.t is balanced by an equal opposite change in the direction of another orbit, the balance of motor direction and energy in nature being exactly preserved.

If such a line of motion pursues a cometary ellipse and enters the atmosphere of a globe, it must be affected by friction precisely as if the line of moving particles were a single particle, or a minute comet. It might be obliterated by friction or resistance, as the orbital motion of a falling body is obliterated. But this obliteration is really caused by the opposing energy of opposite lines of molecular motion. The sincle line of motion may be distributed into a thousand lines differing in direction, but the component of these thousand lines must agree with the original line.

The transfer of motion from particle to particle here indicated may take place through attractive resistance as well as through impact resistance. The original disintegration of the matter of space must have increased, as spheral condensation denuded space of much of its material, and as radiation from the spheres increased its motor energy. If matter thus divided up into smaller and smaller particles, these may have continued as closely contiguous in space as are the molecules of spheral atmospheres. In such a case they may present the conditions of excessive rarity so far as weight of matter is concerned; of close contiguity of particles, sufficient to permit the exercise of attractive energy ; of great compression, through their vigour of centrifugal motion, and of intense elastic resistance to compression. These are the conditions necessary for the transfer of the radiations of light and heat. In these radiatures motion is conveyed through space by transfer of vibratory motions, not of impacts. The vibrating particle swings between lateral chains of attraction, and causes a like transverse swing in successive particles with which it is attractively connected. Greater energy here causes only greater width of vibration, not greater rapidity of transfer. The latter depends only on the elasticity of the matter concerned. Impact transfer of motion, on the contrary, must differ in speed with every difference in vigour. It is transferred by the motions of what we know as local heat, similar to the incessantly varied heat motions of gaseous matter. As the particles are unvarying in weight, increased momentum can be gained only by increased rapidity of motion, and the lines of motion thus transferred through space vary in speed with every variation in vigour. Every motion, of every particle of matter, is really a minute portion of an orbit, which represents that of a falling body, of a planet, or of a comet, according to its rapidity. Though the momentum affects successive particles of matter the orbit is continous, except to the extent that it is varied by perturbations through attraction and impact.

Wherever any influence aids a translation of interspheral matter - causes a wind to blow through space-the lines of motion continue to be conveyed by the same particles. The orbital motions of the spheres are such winds through space; minor aggregations of moving matter may enter the itmosphere of the sun or other globes. But no atmosphere can become permanently increased in this manner; such masses, checked by friction, must yield motion, which flows outward. The centrifugal energy of the molecules of the external atmosphere is thereby increased, and the gain of matter must be balanced by an equal escape of matter at that critical atmospheric limit where centrifugal and centripetal energies are in balance. But any such fall of interspheral matter must aid the radiant emissions of the sun. Its loss of proper motion, its high degree of absolute heat, its increased temperature through condensation, and its consequent radiation, would make it a source of solar heat. Any such cometary matter must form part of "The Fuel of the Sun."

Philadelphia, U.S.

Charles MORris

\section{THE INSTITUTION OF MECHANICAL ENGINEERS}

THE Annual General Meeting of this Institution was held on January 25 and 26 , at the Institution of Civil Engineers, Great George Street; and the papers read were of unusual interest, from a scientific point of view, for a society whose aims are so distinctly practical. As it was pointed out by the president, Mr. Westmacott, three out of the five papers on the list were contributed by professors of science, and dealt with aspects more or less theoretical of the subjects treated upon. This forms, in fact, an additional instance of the way in which the old barriers between theory and practice are breaking down, and it is everywhere bec sming recognised that neither can flourish without the aid of the other.

The first two papers, though quite independent, were both evolved, as it were, out of the same subject, namely, the research which the Institution has for some time been carrying on into the properties of hardencd and unhardened steel. The first of these is an interim report by Prof. Abel, C. B., F.R.S., on the present stage of his experiments relating to the condition in which carbon exists in steel. Preliminary trials had shown that the treatment of steel and iron by a chromic acid solution (produced by mixing a solution of potassium bichromate, saturated in the cold, with one-twentieth of its volume of pure concentrated sulphuric acid) gave great promise of success in detecting the chemical differences existing in the same steel, according to the treatment to which it has been subjected. When cold-rolled, and annealed steel was thus treated, it yielded considerable amounts of an insoluble residue, consisting of black spangly particles, strongly attracted by the magnet, and presenting the characteristics of a true carbide, to which was assigned provisionally the formula $\mathrm{Fe}_{6} \mathrm{C}_{5}$. With hardened steel, on the other hand, but a small quantity of such particles were obtained, mixed with a lighter sediment; and the total residue contained only about one-sixth the carbon in the original steel, whereas in the annealed samples nearly all the original carbon was detected in the residue. The theory to which this points clearly is that in soft steel the carbon exists in a state of chemical combination, forming a carbide which is disseminated as a separate body through the mass of the iron; but that in hard steel this combination is dissolved, and the carbon exists in its pure form, either merely in mechanical admixture, as in the case of grey cast-iron, or in that peculiar and not very well understood form of association which metallurgists term an alloy. It would follow that the process of tempering, or rapid cosling, does not leave time for the complete formation of the carbide, and that in tempered steel all or some of the carbon still survives in its free or alloyed condition.

The fresh experiments described by Prof. Abel give, on the whole, great support to this theory. Four preparations were made of steel dissolved in chromic acid solution made as above, but of different degrees of strength. In the last only, where the strength was very high, were the results different, showing that the carbide had not been able to resist the oxidising effects of the solution. In the others, a considerable deposit was found, which, after being kept for several days, first in the original and afterwards in a fresh solution, was washed and dried, and then analysed. Another portion of the same was treated with chlorhydric acid, in order to ascertain what proportion would be converted into hydrocarbon. When this proportion was deducted from the whole, the remainder showed a most remarkable uniformity of composition, the percentages of carbon in three experiments being $5^{\circ} 93,5^{\circ} 94$, and $6^{\circ} 00$ respectively. It seems evident that we have here a definite compound, to which Prof. Abel gives the formula $\mathrm{Fe}_{3} \mathrm{C}$. The deviations from this exact composition he accounts for by the presence of a certain amount of water, indicating that a carbo-hydrate had been formed probably as a result of the action on the carbide first separated.

Prof. Hughes's paper, which was illustrated by a series of very 